\title{
Tumor del Estroma Gastrointestinal (TEGI). Un estudio de su frecuencia y perfil de inmunohistoquímica*
}

\author{
Drs. MAYRA PÉREZ R. ${ }^{1}$, KARINA PIÑA-O. ${ }^{1}$ \\ 1 Departamento de Anatomía Patológica del Hospital de Especialidades del Centro Médico Nacional del Noreste, \\ Monterrey, Nuevo León, México.
}

\begin{abstract}
Gastrointestinal stromal tumors. Analysis of 51 cases, Immunohistochemical expression

Background: Gastrointestinal Stromal Tumors (GIST) have a mesenchymal origin and correspond to $1 \%$ of all gastrointestinal tumors. They have a benign behavior in approximately $75 \%$ of cases. They express CD117, CD34, smooth muscle actin, S-100 and desmin, markers that are useful in the differential diagnosis of smooth muscle tumors and those of neurogenic origin. Aim: To report our experience with GIST. Material and Methods: A retrospective, observational study. The pathology reports of GIST in the period 2004-2008 were reviewed. Immunohistochemical expression, pathological grade, mitotic index and histological patterns were reviewed. The medical records of patients were reviewed to obtain age and gender, location, size and presence of metastases. Results: A total of 51 GIST were identified, coming from 21 males and 30 females. Nineteen tumors were located in the small bowel, 18 in the stomach, four in the rectum, two in the colon and in five, the location was not specified. In 28 cases, the pathological pattern was spindle cell, in 12 mixed, in six epithelioid, in three pleomorphic, in one signet ring cell and giant cell in one. Forty nine percent of tumors were of high grade. Metastases were found in the liver in two cases, in the omentum in two and in the spleen, kidney, retroperitoneum and pancreas, in one case each. Two had lymph node involvement. Conclusions: GIST tumor corresponded to a $0.12 \%$ of all pathology reports during the study period. Most tumors in this series were of high grade
\end{abstract}

Key words: GIST, spindle cell, immunohistochemical expression, gastrointestinal tumors.

\section{Resumen}

Introducción: Los Tumores del Estroma Gastrointestinal (TEGI) son de origen mesenquimal comprendiendo el 1\% de todos los tumores GI. Son benignos del 70 a 80\%. Expresan CD117, CD34, actina de músculo liso, S-100 y desmina, marcadores útiles en el diagnóstico diferencial de tumores de músculo liso y tumores de origen neurogénico. Material y Método: Es un estudio retrospectivo y descriptivo. Se revisaron los reportes en el período 2004-2008 registrados como TEGI, valorando la expresión Inmunohistoquímica, grado histológico, índice mitótico, y patrones histológicos. Del reporte histológico se obtuvo la edad y sexo del paciente, localización, tamaño y metástasis. Resultados: Se recolectaron 51 casos corroborados como

*Recibido el 29 de Diciembre de 2009 y aceptado para publicación el 19 de Abril de 2010.

Correspondencia: Dra. Mayra Pérez P.

Ave. Lincoln y Ave. Gonzalitos S/N. Col. Mitras Norte C.P. 64320. Monterrey, Nuevo León, México. E-mail: adierenem@yahoo.com 
TEGI. Encontrando una prevalencia del sexo femenino (30) y una edad media de 52 años. Las localizaciones fueron: Intestino delgado (19), estómago (18), no especificado (5), recto (4), colon (2), retroperitoneo (2), no encontramos en esófago. Los patrones encontrados fueron el fusocelular (28), mixto (12), epitelioide (6), pleomórfico (3), células en anillo de sello (1), células gigantes (1). La mayoría (49\%) fue de alto grado, presentando metástasis a hígado (2), ganglios (2), epiplón (2), bazo (1), riñón (1), retroperitoneo (1) y páncreas (1). Discusión: Se realizaron un total de 41.035 estudios histopatológicos, de los cuales 51 casos corresponden a TEGI, esto equivale al $0,12 \%$. Encontramos tumores en los que su morfología, tamaño e índice mitótico fueron de bajo grado y presentaron metástasis y recidivas al momento del diagnóstico. Veinticinco casos fueron de alto grado (49\%), lo cual es mayor a lo reportado por la literatura $20-30 \%$, probablemente porque este es un hospital de concentración y generalmente los pacientes acuden a atención médica en una etapa avanzada de la enfermedad.

Palabras clave: TEGI, fusocelular, epitelioide, pleomórfico.

\section{Introducción}

En 1984, Schaldenbrand y Appelman, introdujeron el término tumor estromal para referirse a un grupo de neoplasias mesenquimales las cuales previamente eran conocidas con diversos nombres debido a su diferenciación neural o muscular. $\mathrm{Mu}-$ chos de estos tumores demostraron hallazgos típicos de diferenciación en músculo liso y fueron referidos como leiomiomas o leiomiosarcomas ${ }^{1}$. Con el advenimiento de la inmunohistoquímica comenzó su verdadero entendimiento y se introdujo a la modificación de su manejo médico y quirúrgico ${ }^{2}$.

Los tumores del estroma gastrointestinal (TEGI) son los tumores más comúnmente encontrados de origen en el tejido mesenquimal en el tracto gastrointestinal (GI), comprendiendo el $1 \%$ de todos los tumores GI. $\mathrm{Su}$ frecuencia es de 10 a 20 casos por cada millón de personas. Se presentan a cualquier edad (pico en la 5 a a 6 a década). Son benignos del 70 a $80 \%$. La mayoría (60 a 70\%) se encuentran en el estómago, 25 a 35\% se encuentran en el intestino delgado, $5 \%$ en el colon y recto, y $2-3 \%$ en el esófago. Con menor frecuencia, también pueden encontrarse en otros lugares, como en el epiplón, mesenterio o el retroperitoneo ${ }^{2-5}$.

Se consideran de alto riesgo de malignidad aquellos tumores mayores de $5 \mathrm{~cm}$, con un índice mitótico mayor de 5 en 50 campos en alto poder, con una alta celularidad, presencia de necrosis y bordes infiltrantes ${ }^{4}$.

CD117 (c-kit): Es un epítopo del dominio extracelular del receptor de superficie de cinasa de tirosina (c-kit), cuyo ligando es el factor de células tallo (en inglés SCF). Cromosoma 4q12. La activación de c-kit dimeriza al receptor, fosforila e inicia la transducción de la señal química hacia el núcleo para la reproducción y diferenciación celular. Los (TEGI) expresan CD117 en un 85\%; CD34 en un $60-70 \%$, principalmente a nivel de esófago; actina de músculo liso en un $30-40 \%$; $\mathrm{S}-100$ en un $5 \%$ y raramente expresan desmina ${ }^{2-4}$.
También lo expresan: células intersticiales de Cajal, mastocitos, melanocitos, células madre hematopoyéticas y células germinales.

Tumores que lo expresan ocasional y focalmente: Tumor de Ewing, melanoma, angiosarcoma, schwannoma melanótico y condrosarcoma mixoide extraesquelético.

El CD34 es un antígeno encontrado con más frecuencia en células precursoras hematopoyéticas y células endoteliales vasculares, también está presente en las células de los TEGI, pero a un menor grado que el CD117. El lugar de los TEGI primarios puede reflejarse en el porcentaje de positividad de CD34 (es decir, los tumores esofágicos generalmente son más positivos para CD34 que los tumores del intestino delgado). Además, la desmina (una proteína filamentosa intermedia encontrada en las células musculares), la actina de músculo liso (AML), y el S-100 (un antígeno celular de cresta neural) también son útiles en el diagnóstico diferencial de TEGI $\mathrm{y}$ ayudan a diferenciar tumores de músculo liso $\mathrm{y}$ tumores de origen Neurogénico ${ }^{2-4}$.

\section{Material y Método}

Es un estudio retrospectivo y descriptivo. Se revisaron los reportes histopatológicos de los archivos de patología quirúrgica del Hospital de Especialidades No. 25 CMN Noreste, IMSS, Monterrey registrados en el período de 2004-2008 y que corresponde a 51 casos, seleccionando todos aquellos casos que reúnen cualquiera de las siguientes características:

1. Tumores diagnosticados como TEGI.

2. Neoplasias clasificadas como de origen neural de cualquier parte del tubo digestivo.

3. Neoplasias clasificadas como de origen muscular, originadas en cualquier parte del tubo digestivo.

4. Neoplasias fusocelulares o epitelioides con proximidad anatómica al tubo digestivo, bien sean del retroperitoneo, mediastino, cuello, etc. 


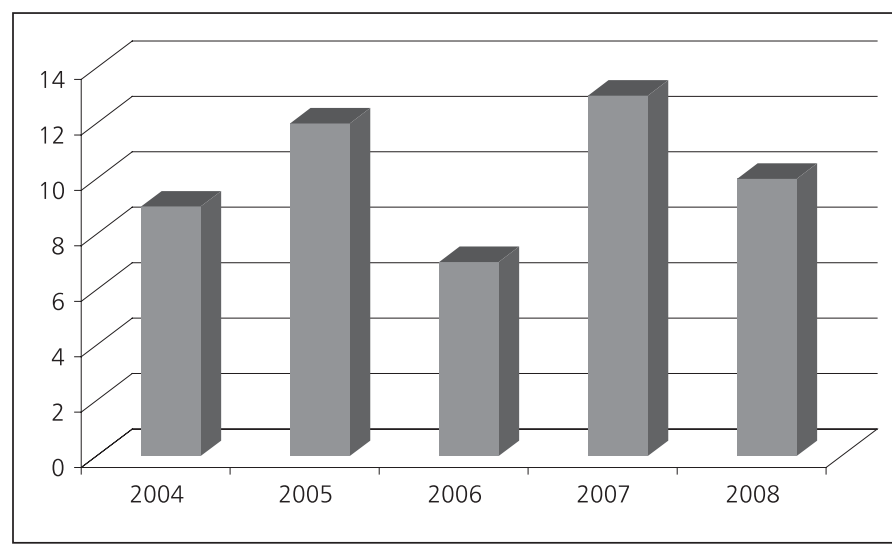

Figura 1. Casos por año de Tumor del Estroma Gastrointestinal (GIST) en el período de 2004 a 2008, en el Hospital N 25 del IMSS Mty N.L.

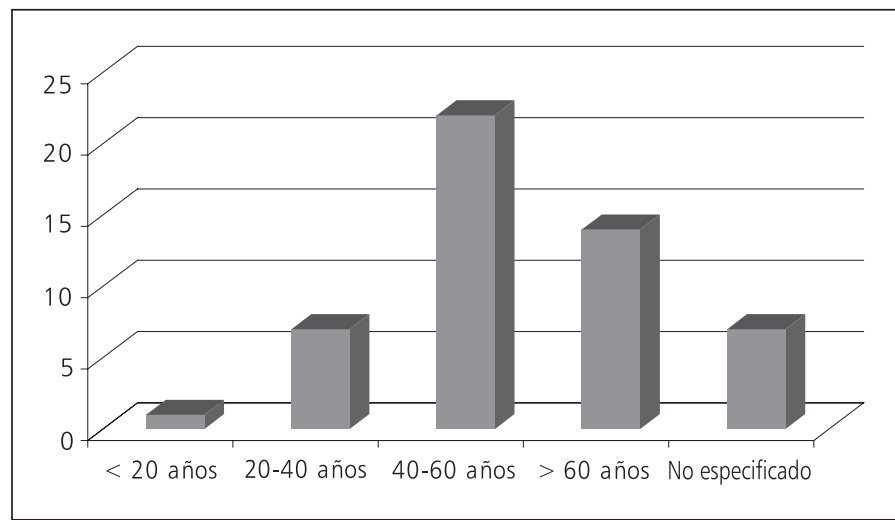

Figura 2. Distribución por edad de los Tumores del Estroma Gastrointestinal (GIST) en el período de 2004 a 2008, en el Hospital $N^{\circ} 25$ del IMSS Mty N.L.

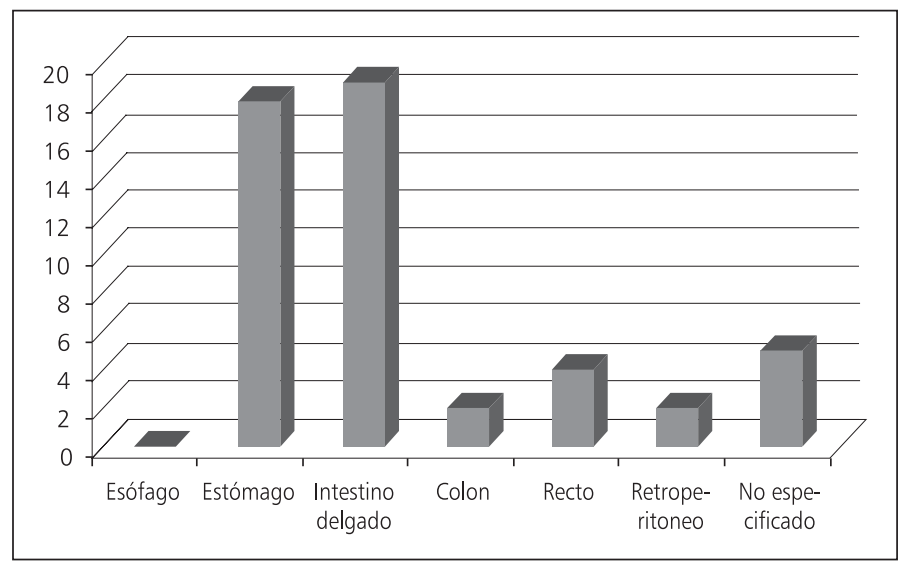

Figura 3. Localizaciones más frecuentes encontradas en los Tumores del Estroma Gastrointestinal (GIST) en el período de 2004 a 2008, en el Hospital N² 25 del IMSS Mty N.L.
Se recolectaron las laminillas histológicas, las cuales se revisaron y se localizaron los bloques de parafina para recorte con tinciones de rutina para demostrar utilidad y preservación de los tejidos y subsecuentemente se obtuvieron recortes de $6 \mu \mathrm{m}$ de espesor para inmunohistoquímica de CD117, CD34, proteína $\mathrm{S}-100$ y desmina. Con la metodología $\mathrm{LSAB}+$.

Se valoró la expresión Inmunohistoquímica de los diferentes inmunorreactantes mencionando su positividad tomando en cuenta las células teñidas en su citoplasma y su membrana citoplásmica, en escala de 1 a 4+ en cuanto a su intensidad de tinción, siendo $1+$ interpretado como bajo, $2+$ moderado, $3+$ intenso y 4+ muy intenso; así como la proporción semicuantitativa en porcentaje de las células neoplásicas positivas respecto de las negativas en escala de 0 a $100 \%$, se valoró el tamaño de acuerdo a la clasificación del riesgo de los tumores estromales de acuerdo con su tamaño. Del reporte histológico se obtuvieron los siguientes datos: Edad y sexo del paciente, ubicación-topografía de la neoplasia, tamaño de la misma, presencia o no de enfermedad metastásica al momento del reporte original.

De las laminillas originales o recortes teñidos con H-E se evaluó el grado histológico, índice mitótico, y patrones histológicos presentes, además de los ya conocidos (epitelioide y fusocelular), se buscó y clasificó otros patrones con los que cuenta este tumor.

\section{Resultados}

En un período de cinco años, comprendidos entre el 2004 y el 2008, se recolectaron $9,12,7,13$ y 10 casos por año respectivamente, diagnosticados y corroborados como TEGI, con un total de 51 casos en cinco años. Figura 1.

En un período de cinco años (20042008) se realizaron un total de 41.035 estudios histopatológicos, de los cuales sólo el $0,12 \%(f=51)$ correspondieron a Tumores del Estroma Gastrointestinal.

Se encontró una prevalencia del 


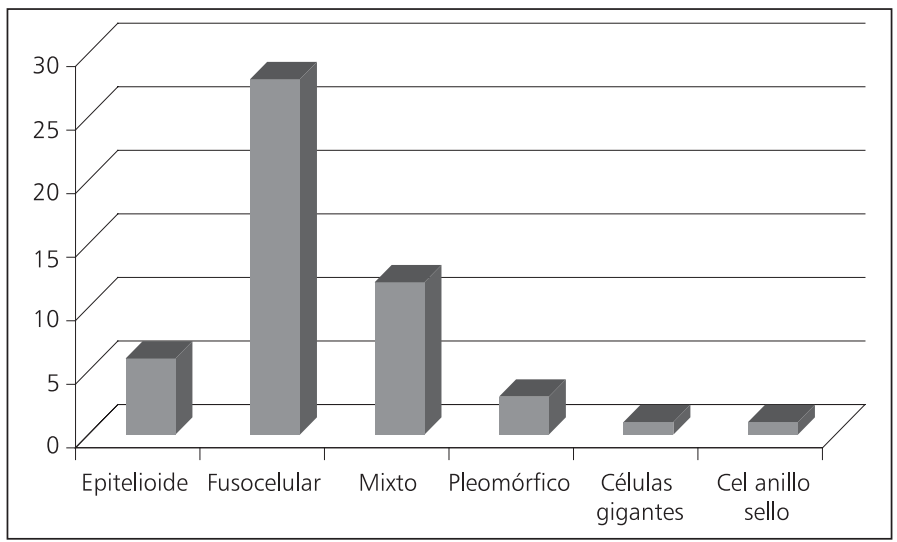

Figura 4. Frecuencia de los patrones histopatológicos encontrados en los TEGI.

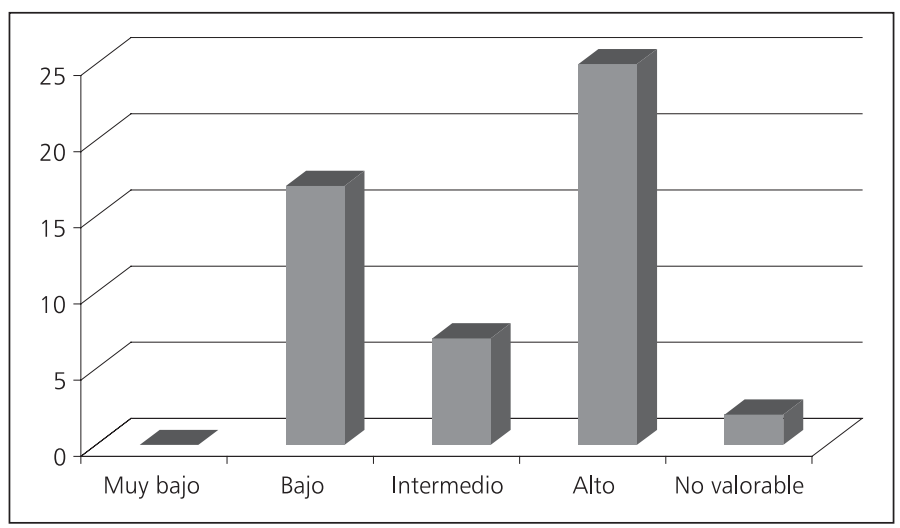

Figura 5. Grados de malignidad encontrados en los casos de Tumores del Estroma Gastrointestinal (GIST) en el período de 2004 a 2008, en el Hospital $N^{\circ} 25$ del IMSS Mty N.L.

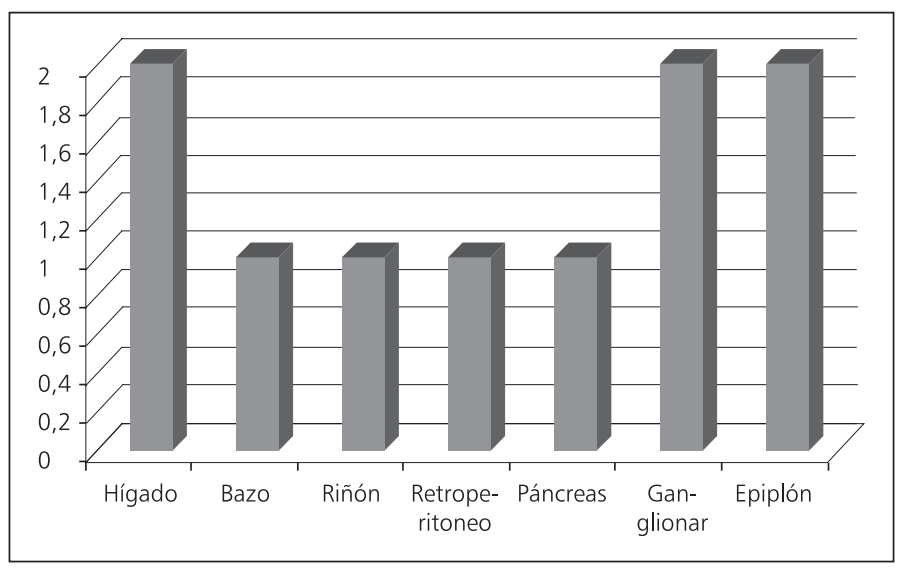

Figura 6. Sitios de metástasis encontradas en los Tumores del Estroma Gastrointestinal (GIST) en el período de 2004 a 2008, en el Hospital $\mathrm{N}^{\circ} 25$ del IMSS Mty N.L. sexo femenino $(\mathrm{n}=30)$, respecto al sexo masculino $(\mathrm{n}=21)$.

La distribución de edad fue desde los 19 años (1), de 20 a 40 años (7), de 40 a 60 años (22), > 60 años (14) y con edad no especificada en el reporte histopatológico (7), con una media de 52 años. Figura 2.

Las localizaciones más frecuentes fueron en orden de frecuencia: Intestino delgado (19), estómago (18), sitio no especificado (5), recto (4), colon (2), retroperitoneo (2) y esófago (0). Figura 3.

El patrón que predominó fue el fusocelular $(\mathrm{n}=28$, seguido por el mixto $(n=12)$, el epitelioide $(n=6)$, pleomórfico $(\mathrm{n}=3)$, células en anillo de sello $(n=1)$, células gigantes $(n=$ 1). Figura 4.

La mayoría de los casos correspondió a TEGI de alto grado $(\mathrm{n}=25)$, seguido por bajo grado $(n=17)$, grado intermedio $(n=7)$, y 2 casos con grado no valorable por que el tejido era muy escaso. Figura 5.

Las metástasis encontradas al momento del diagnóstico se aprecian en la Figura 7.

Respecto a los resultados de inmunohistoquímica, el CD-117 fue positivo en el 90,19\% $(\mathrm{n}=46)$, el CD34 resultó positivo en un $60,78 \%(n=31)$, la actina en un $35,29 \%(\mathrm{n}=18)$, la desmina en un $49,01 \%(\mathrm{n}=25)$, y la proteína $\mathrm{S}-100$ en un $41,17 \%(\mathrm{n}=21)$.

\section{Discusión}

Entre los años 2004 y 2008, se realizaron un total de 41.035 estudios histopatológicos, de los cuales 51 casos corresponden a TEGI, esto equivale al $0,12 \%$.

Su localización más frecuente está en intestino delgado. Figuras 7 y 8 .

Existen TEGI negativos para el CD 117 , por lo que es necesario investigar su positividad para el CD 34 para corroborar su diagnóstico. También es importante realizar inmunohistoquímica para descartar otro tipo de lesiones con las que se realiza en diagnóstico diferencial de estos tumores.

Con nivel de evidencia 2 (no man- 


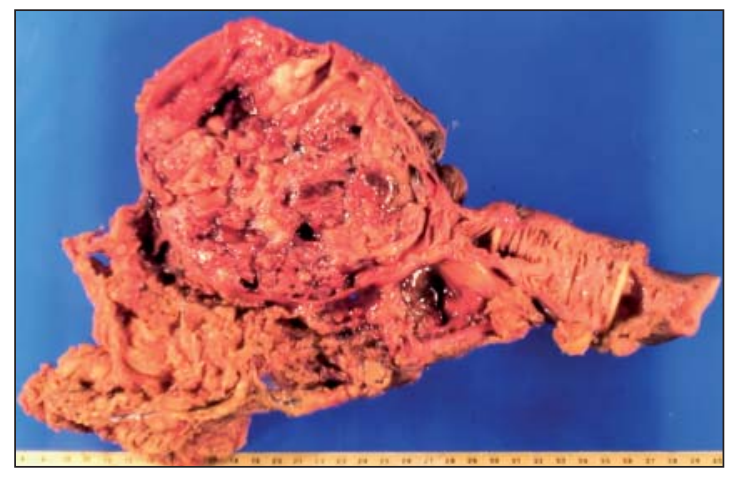

datario), ningún TEGI debiera ser considerado benigno $^{6}$ ya que encontramos tumores en los que su morfología, tamaño e índice mitótico fueron de bajo grado y presentaron metástasis y recidivas al momento del diagnóstico. Gracias a los avances de la inmunohistoquímica se ha podido diferenciar este tipo de neoplasias para clasificarlas mejor y poder dar un diagnóstico definitivo, por lo que el patólogo tiene la obligación de reportar todas sus características ya descritas para administrar el tratamiento de elección.

En cuanto al grado de malignidad, 25 casos fueron de alto grado $(49 \%)$, lo cual es mayor a lo reportado por la literatura (20-30\%), esto es debido a que este es un hospital de concentración y generalmente los pacientes acuden a atención médica en una etapa avanzada de la enfermedad.

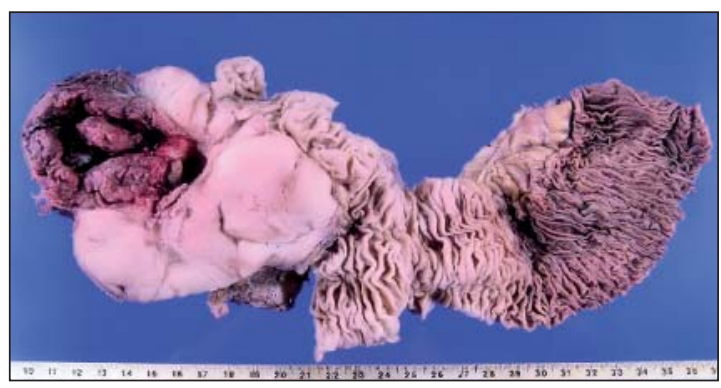

Figura 7 y 8. GIST de localización más frecuente en intestino delgado.

\section{Referencias}

1. Thomas RM, Sobin LH. Gastrointestinal Cancer incidence and by histologic type, SEER population-based data 1973-1987. Cancer 1995; 75: 154-170.

2. Dávila R, Fardel D. GI Stromal tumors. Gastrointest Endosc 2003; 58: 80-88.

3. Robbins y Cotran. Tumores de la estroma gastrointestinal. Patología Estructural y Funcional. 7ª Edición Elsevier; 2006: 830-831.

4. Rosai J. GIST. Ackerman's Surgical Pathology. Vol 1. $9^{\text {th }}$ Edition Mosby; 2004: pp. 632, 674-680, 734-735, $769,822-823$.

5. Domínguez LC, Torregosa L. Tumores gástricos estromales. Rev Colomb Cir 2003; 18: 110-115.

6. Eizaguirre B, Burgos JJ. Rev Esp Patol 2006; 39: 209218. 